\title{
Melatonin induces transcriptional regulation of Bim by FoxO3a in HepG2 cells
}

\author{
S Carbajo-Pescador ${ }^{1}$, C Steinmetz ${ }^{2}$, A Kashyap ${ }^{2}$, S Lorenz ${ }^{2}$, J L Mauriz ${ }^{1}$, M Heise ${ }^{3}$, P R Galle ${ }^{2}$, \\ J González-Gallego ${ }^{1}$ and S Strand ${ }^{\star, 2}$
}

${ }^{1}$ Centro de Investigación Biomédica en Red de Enfermedades Hepáticas y Digestivas (CIBERehd) and Institute of Biomedicine, University of León, León, Spain; ${ }^{2}$ Department of Internal Medicine, University Medical Center, Johannes Gutenberg University, Mainz, Germany and ${ }^{3}$ Department of Transplantation and Hepatobiliary Surgery, University Medical Center, Johannes Gutenberg University, Mainz, Germany

Background: Melatonin induces apoptosis in many different cancer cell lines, including hepatocellular carcinoma cells. However, the responsible pathways have not been clearly elucidated. A member of the forkhead transcription factors' family, FoxO3a, has been implicated in the expression of the proapoptotic protein Bim (a Bcl-2-interacting mediator of cell death). In this study, we used human HepG2 liver cancer cells as an in vitro model to investigate whether melatonin treatment induces Bim through regulation by the transcription factor FoxO3a.

Methods: Cytotoxicity of melatonin was compared in HepG2 hepatoblastoma cells and primary human hepatocytes. Proapoptotic Bim expression was analysed by reverse transcriptase-polymerase chain reaction and western blot. Reporter gene assays and chromatin immunoprecipitation assays were performed to analyse whether FoxO3a transactivates the Bim promoter. Small interfering RNA (siRNA) was used to study the role of FoxO3a in Bim expression. Immunofluorescence was performed to analyse FoxO3a localisation in HepG2 cells.

Results: Melatonin treatment induces apoptosis in HepG2 cells, but not in primary human hepatocytes. The proapoptotic effect was mediated by increased expression of the BH3-only protein Bim. During melatonin treatment, we observed increased transcriptional activity of the forkhead-responsive element and could demonstrate that FoxO3a binds to a specific sequence

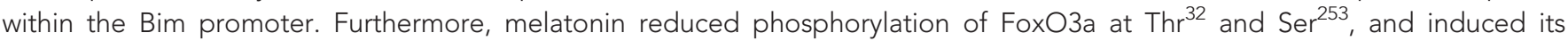
increased nuclear localisation. Moreover, silencing experiments with FoxO3a siRNA prevented Bim upregulation.

Conclusion: This study shows that melatonin can induce apoptosis in HepG2 hepatocarcinoma cells through the upregulation of proapoptotic Bim mediated by nuclear translocation and activation of the transcription factor FoxO3a.

Each year, hepatocellular carcinoma (HCC) is diagnosed in more than half a million people worldwide, being the most common malignant hepatobiliar disease (El-Serag, 2011; Jemal et al, 2011). Viral hepatitis B and C, as well as alcohol abuse, are the main risk factors for its development (Cornella et al, 2011). Dysregulation of cellular proliferation and apoptosis are frequent events related with malignant phenotype and poor responsiveness of HCC towards chemotherapy (Müller et al, 1997). For this reason, advances in understanding these processes are needed for developing effective pharmacological therapies for HCC.
Melatonin, the hormone of the pineal gland, controls circadian rhythms, and it has been reported to exert additional functions in other organs. A large number of studies have demonstrated the protective role of melatonin in different pathophysiological situations in the liver, showing antioxidant and antiapoptotic proprieties (Pan et al, 2006; Subramanian et al, 2007; Thong-Ngam et al, 2007; Tahan et al, 2009). On the other hand, in vitro studies with different cancer cell lines have provided evidence for melatonin induction of apoptosis in tumour cells (Hill and Blask, 1988; Farriol et al, 2000; Futagami et al, 2001; Cini et al, 2005;

*Correspondence: Dr S Strand; E-mail: sstrand@uni-mainz.de

Received 23 July 2012; revised 13 November 2012; accepted 17 November 2012; published online 20 December 2012

(c) 2013 Cancer Research UK. All rights reserved 0007-0920/13 
Garcia-Santos et al, 2006; Garcia-Navarro et al, 2007; Cabrera et al, 2010; Chiu et al, 2010; Gonzalez et al, 2010). We have recently reported that melatonin administration induces cell cycle arrest and apoptosis in hepatocarcinoma HepG2 cells through MT1 melatonin receptor by modulation of cAMP basal levels and ERK kinase activation (Carbajo-Pescador et al, 2009, 2011). Furthermore, melatonin-induced apoptosis was related with enhanced caspase- 3 and caspase- 9 activity, cytosolic cytochrome $c$ release and upregulation of the proapoptotic protein Bax (Martin-Renedo et al, 2008). Nevertheless, the molecular pathways that underlie melatonin-induced apoptosis in human HCC are not fully elucidated.

The FoxO subfamily of forkhead transcription factors (FoxO1/ FKHR, FoxO3/FKHRL1 and FoxO4/AFX identified in mammals) plays an important role in tumour suppression by upregulating target genes involved in cell cycle arrest and apoptosis. Interestingly, low levels of FoxO3 have been reported to confer chemotherapy resistance in human cancers, being significantly associated with poor prognosis in cancer patients (Jin et al, 2004; Fei et al, 2009; Su et al, 2011). Moreover, enhanced activity and expression of active forms of FoxO transcription factors is required for tumour chemosensitisation (Sunters et al, 2003; Paroni et al, 2011). FoxO proteins are activated in response to a wide range of external stimuli. Regulation of its activity depends mainly on changes in the subcellular localisation, achieved via post-translational modifications, including phosphorylation, acetylation and ubiquitination (Calnan and Brunet, 2008). Several genetic and biochemical studies indicate that the FoxO family is a key downstream target of the PI3K-Akt pathway in development and longevity (Lin et al, 1997; Brunet et al, 1999). Thus, phosphorylation of FoxO factors in specific serine and/or threonine sites modulates their subcellular localisation (Rena et al, 2002; Barthel et al, 2005; Anton et al, 2007). Once placed in the nucleus, they play tumour suppressor roles through enhanced transcription of proapoptotic genes, such as $B C L 6$, a Bcl-2-interacting mediator of cell death (Bim), and Fas ligand (Dijkers et al, 2000; Yang et al, 2006).

$\mathrm{Bim}$ is a proapoptotic member of the Bcl-2 family, and is one of the main downstream targets of FoxO3a. After transcription, Bim mRNA undergoes an alternate splicing, giving three isoforms (BimS, BimL and BimEL) with different length (Ewings et al, 2007). Interestingly, there are in vivo and in vitro evidence demonstrating an essential role of Bim proteins in Bax activation (Ren et al, 2010). Based on this information, we focused this study on the FoxO3a regulation of Bim expression after treatment with pharmacological concentrations of melatonin, in an attempt to gain further mechanistic insights on the molecular pathways leading to melatonin-induced apoptosis in HepG2 liver cancer cells.

\section{MATERIALS AND METHODS}

Cell culture. HepG2 cells were obtained from the American Type Culture Collection (Manassas, VA, USA) and grown in Dulbecco's modified Eagle's medium (DMEM). Primary human hepatocytes were isolated from healthy liver tissue of patients undergoing partial hepatectomy by two-step collagenase perfusion. Cells were seeded on collagen-coated culture dishes in Williams medium supplemented with $10 \%$ fetal bovine serum, $15 \mathrm{mmoll}^{-1}$ HEPES ( $\mathrm{pH} 7.4$ ), $2 \mathrm{mmoll}^{-1}$ glutamine, $100 \mathrm{Uml}^{-1}$ penicillin and $100 \mu \mathrm{g} \mathrm{ml}^{-1}$ streptomycin. LY294002 was from Tocris (Bristol, UK). Melatonin and epidermal growth factor (EGF) were obtained from Sigma-Aldrich (St Louis, MO, USA).

Viability assays. HepG2 cells or primary human hepatocyte were seeded in 96-well plates. Melatonin dissolved in dimethyl sulphoxide (DMSO) was added to the cells at the concentrations as indicated in the figures. Apoptosis was induced in HepG2 cells with $200 \mathrm{ng} \mathrm{ml}^{-1}$ of the monoclonal antibody (Ab) to human (APO-1/Fas) anti-APO-1, kindly provided by Peter $\mathrm{H}$ Krammer. Cell viability was determined using the CellTiter-Glo (Promega, Fitchburg, WI, USA) and 3-(4,5-Dimethythiazol-2-yl)-2,5-diphenyl tetrazolium bromide (MTT) assays (Sigma-Aldrich). CellTiterGlo assay was performed according to the manufacturer's instructions (Promega). Luminescence was determined in a Saphire luminometer (Tecan Austria, Grödig, Austria). The MTT assay was carried out as described by Denizot and Lang (1986). Briefly, after exposure of cells to melatonin, culture media were changed by serum-free culture media. 3-(4,5-Dimethythiazol-2-yl)2,5-diphenyl tetrazolium bromide dissolved in phosphate-buffered saline (PBS) was added to each well for $3 \mathrm{~h}$. After this interval, the culture media containing MTT were discarded and DMSO was added to each well, dissolving the precipitate. The optical densities were measured at $560 \mathrm{~nm}$ spectral wavelength using microtitre plate reader (Synergy HT Multi-Mode Microplate Reader; Bio-Tek Instruments Inc., Winooski, VT, USA).

Transfection and luciferase reporter assay. Transient transfection of HepG2 human hepatocytes was performed using the TransFectin reagent (Bio-Rad, Hercules, CA, USA). Constructs used were the FHRE-Luciferase reporter (Addgene plasmid 1789 kindly provided by M Greenberg's lab) (Tran et al, 2002) and the FoxO3a expression construct (Addgene plasmid 8355 kindly provided by A Brunet's lab) (Brunet et al, 1999). Inducible activation of FoxO3a was performed through transfection of the HA-FoxO3a-WT-ER plasmid. The HA-FoxO3a-WT-ER fusion protein is constitutively expressed but is inhibited unless exposed to a modified ligand for the oestrogen receptor (ER), 4-hydroxytamoxifen (4-OHT). HepG2 cells were transfected using the TransFectin reagent (Bio-Rad, Munich, Germany) with $1 \mu \mathrm{g}$ of HA-FoxO3a-WT-ER plasmid (Tran et al, 2002). Activation of the accumulated FoxO3a protein was induced by treatment with the ER ligand 4-OHT $1 \mathrm{~h}$ before melatonin treatment. The luciferase reporter activity was measured using a commercially available luciferase assay system (Promega). Transfection efficiency was normalised by $\beta$-galactosidase activity.

Western blot analysis. After treatments, cultured cells were washed two times with ice-cold PBS and lysed by adding ice-cold RIPA buffer containing $50 \mathrm{~mm}$ Tris- $\mathrm{HCl}$ (pH 7.4), $150 \mathrm{~mm} \mathrm{NaCl}$, 2 mm EDTA, $0.1 \%$ Triton X-100, 10\% sodium deoxycholate, $10 \%$ SDS, $1 \mathrm{~mm} \mathrm{NaF}$ and protease cocktail inhibitor (Roche, Basel, Switzerland), and scraped off the plate. The extracts were transferred to a microfuge tube and centrifuged for $10 \mathrm{~min}$ at $15000 \mathrm{~g}$. Equal amounts of the supernatant protein $(20 \mu \mathrm{g})$ were separately subjected to SDS-PAGE and transferred to a PVDF membrane (Bio-Rad). Primary Abs were diluted in blocking solution and incubated overnight at $4{ }^{\circ} \mathrm{C}$ with polyclonal $\mathrm{Ab}$ to Bim (rabbit, 1:1000 dilution; eBioscience, San Diego, CA, USA), phospho-FoxO3a $\mathrm{Thr}^{32}$ and FoxO3a Ser ${ }^{253}$ (rabbit, 1:1000 dilution; Cell Signalling Technology, Beverly, MA, USA) and FoxO3a (rabbit, 1:1000 dilution; Abcam, Cambridge, UK). Equal loading of protein was demonstrated by probing the membranes with a rabbit anti- $\beta$-actin polyclonal Ab (1:2000 dilution; Sigma), anti-lamin B1 (H-90) (1:2000 dilution; Santa Cruz Biotechnology, Santa Cruz, CA, USA) or anti-tubulin (Sigma-Aldrich). After washing with PBST, membranes where incubated with phosphatase-conjugated anti-rabbit secondary Ab from Sigma-Aldrich diluted in blocking solution and incubated for $1 \mathrm{~h}$ at room temperature. The proteins were detected and visualised by chemiluminescence using the CDP star detection system (Tropix Applied Biosystems, Carlsbad, CA, USA). Densitometry analysis of specific bands was performed by the Scion Image software (Scion Corporation, Frederick, MD, USA). 
Real-time reverse transcriptase-polymerase chain reaction. For real-time reverse transcriptase-polymerase chain reaction (RTPCR), confluent HepG2 cells growing in complete media were replated in six-well culture plates, at a density of 150000 cell per well in a total volume of $2 \mathrm{ml}$ of complete medium. After treatment, total RNA was obtained by using a Trizol reagent (Applied Biosystems, Carlsbad, CA, USA) and quantified by spectrophotometry (Nanodrop 1000; Thermo Scientific, Waltham, MA, USA). The iScript cDNA Synthesis Kit (Bio-Rad) was used to reverse transcribe RNA into cDNA. Real-time PCR was performed using the iCycler Absolute QPCR SYBR Green Mix (ABgene, Waltham, MA, USA). Bim mRNA levels were normalised to RNA polymerase II (RpII) using the $2^{-\Delta \mathrm{Ct}}$ method based on the threshold cycle (CT) value. Primer sequences were as follows: Bim, $5^{\prime}$-AACCACTATCTCAGTGCAAT- $3^{\prime}$ and $5^{\prime}$-GGTCTTCGGCTG CTTGGTAA-3'; RPII, 5'-GCACCACGTCCAATGACAT- $3^{\prime}$ and 5'-GTGCGGCTGCTTCCATAA-3'.

Small interfering RNA transfection. HepG2 cells $\left(0.5 \times 10^{6}\right.$ cells per $\mathrm{ml}$ ) were seeded in DMEM medium without antibiotics overnight. After washing the cells with PBS, $1 \mathrm{ml}$ of media without antibiotics were added. Thereafter, $200 \mu \mathrm{l}$ of Lipofectamine 2000 complex was added into each plate. The cells were transfected with FoxO3a small interfering (siRNA) (FKHRL1 siRNA sc-37887) and Bim siRNA (sc-29802) (Santa Cruz Biotechnology) for $48 \mathrm{~h}$ according to the manufacturer's instructions. A non-targeting siRNA-A sc-37007 was used as a negative control. At $48 \mathrm{~h}$ after transfection, medium was replaced for complete DMEM and cells were treated with or without melatonin.

Chromatin immunoprecipitation assays. Chromatin-immunoprecipitation (ChIP) assays were performed using chromatin immunoprecipitation kit (Upstate Cell Signaling, Lake Placid, NY, USA) according to the manufacturer's instructions. Samples treated with the $1000 \mu \mathrm{m}$ melatonin concentration were immunoprecipitated with anti-FoxO3a Ab (Abcam) or rabbit IgG (Sigma). Polymerase chain reaction was performed using primers specific for the Bim promoter: forward, 5'-CCTTCGCGAGGACCAACCCAGTC-3' and reverse, 5'-CCGCTCCTACGCCCAATCACTGC3'.

Immunofluorescence. HepG2 cells were seeded in eight-well chamber slides and then treated with melatonin as indicated. After treatment, they were fixed in $4 \%$ paraformaldehyde and stained with Abs to FoxO3a (rabbit, 1:100 dilution; Abcam). Alexa Fluor 488-labelled anti-rabbit Ab (Invitrogen, Carlsbad, CA, USA) was used as a secondary $\mathrm{Ab}$. Counterstaining of nuclei was performed with Hoechst 33342 (Invitrogen). The cells were imaged directly in the chambers using a Zeiss LSM 710 NLO confocal laser scanning microscope and image analysis was performed using the Zeiss Zen-2009 software (Carl Zeiss Microimaging GmbH, Jena, Germany).

Statistical analysis. Results are expressed as mean values \pm s.e.m. of the indicated number of experiments. A $t$-test was used to determine differences between pairs of treatments, as indicated in Results. One-way ANOVA followed by Student-Newmann-Keuls post hoc test was used to determine differences between the mean values of the different treated groups. $P<0.05$ was considered significant. Values were analysed using the statistical package Statistica 10.0 (Statsoft Inc., Tulsa, OK, USA).

\section{RESULTS}

Effect of melatonin treatment on cell viability. Most types of antitumour therapy result in a certain amount of damage to healthy tissues with associated side effects. Previously, we have shown that melatonin has oncostatic effects in HepG2 liver cancer
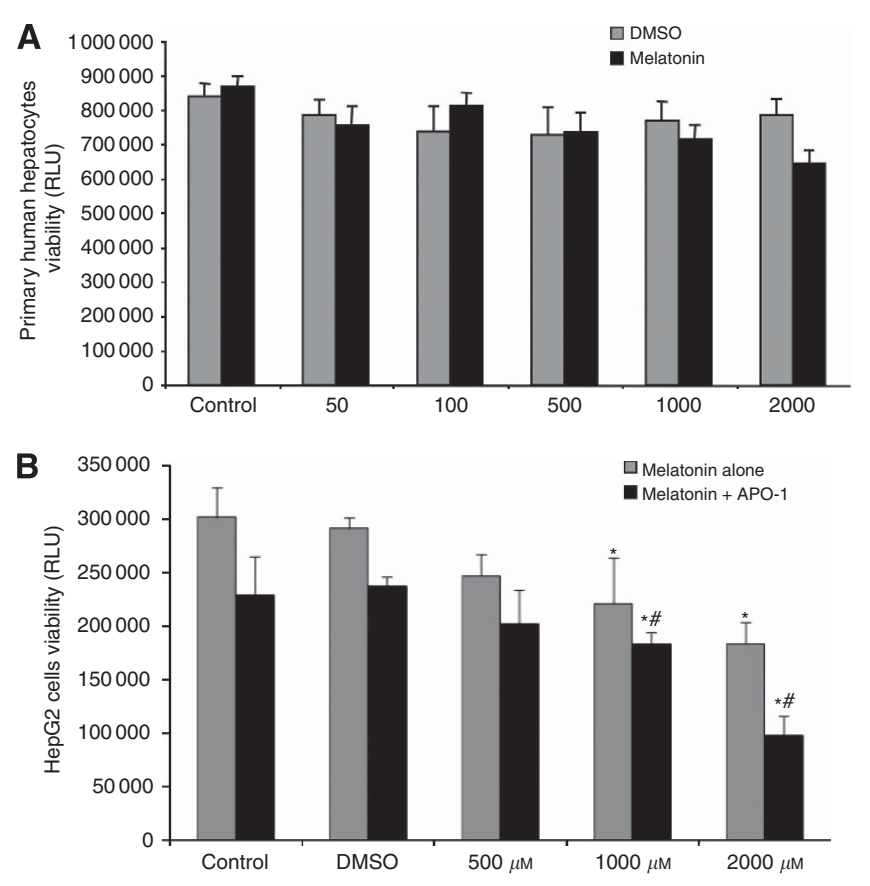

Figure 1. Effect of melatonin treatment on cell viability in primary human hepatocytes (A) and HepG2 cells (B). Data are expressed as a percentage of mean values \pm s.e.m. of four experiments performed in triplicate. ${ }^{\star} P<0.05$ significant differences vs control. ${ }^{*} P<0.05$ significant differences between melatonin and melatonin + APO-1treated cells. Abbreviations: RLU = relative light unit.

cells, and in this study, we used healthy primary human hepatocytes to investigate the selectivity of melatonin between healthy and cancerous cells. In our experiments, $48 \mathrm{~h}$ melatonin treatment from 50 to $2000 \mu \mathrm{M}$ did not significantly affect the viability of primary human hepatocytes (Figure 1A). In contrast, growth inhibition of HepG2 cancer cells under melatonin treatment was dose-dependent (40\% reduction vs control), becoming even higher following preincubation with the human apoptosis inductor anti-APO-1 (60\% reduction $v s$ control) (Figure 1B). The melatonin concentration that exerted the strongest growth inhibition (1000 and $2000 \mu \mathrm{M})$ in HepG2 cells was used in further experiments. These results suggest that melatonin selectively protects normal primary human hepatocytes from injury during apoptosis induction. Next, we focused on elucidating the molecular pathway leading to the proapoptotic effects of melatonin in liver cancer cells.

Effect of melatonin treatment on FoxO3a transcriptional activity. FoxO transcription factors play an important role in tumour suppression. To determine whether FoxO3a was activated by melatonin treatment, HepG2 cells were transfected with a luciferase reporter constructs containing FoxO3a response element. Following a kinetic experiment from 1 to $48 \mathrm{~h}, 1000 \mu \mathrm{M}$ melatonin incubation increased FoxO3a activity with values that represent approximately $150 \%$ of control after 24 and $48 \mathrm{~h}$. Moreover, luciferase activities were more elevated with a higher concentration of $2000 \mu \mathrm{M}$, reaching a maximum of $173 \%$ at $48 \mathrm{~h}$ compared with control (Figure 2A).

Effect of melatonin treatment on Bim expression. After analysing transactivation of FoxO3a-responsive promoter elements after melatonin treatment, we studied the $\mathrm{BH} 3$-only protein of the Bcl-2 family Bim, a known downstream target of FoxO3a, required for the initiation of apoptosis. Both melatonin concentrations tested increased Bim EL protein level, with maximum values reached at 24 and $48 \mathrm{~h}$. Western blot results were consistent with 

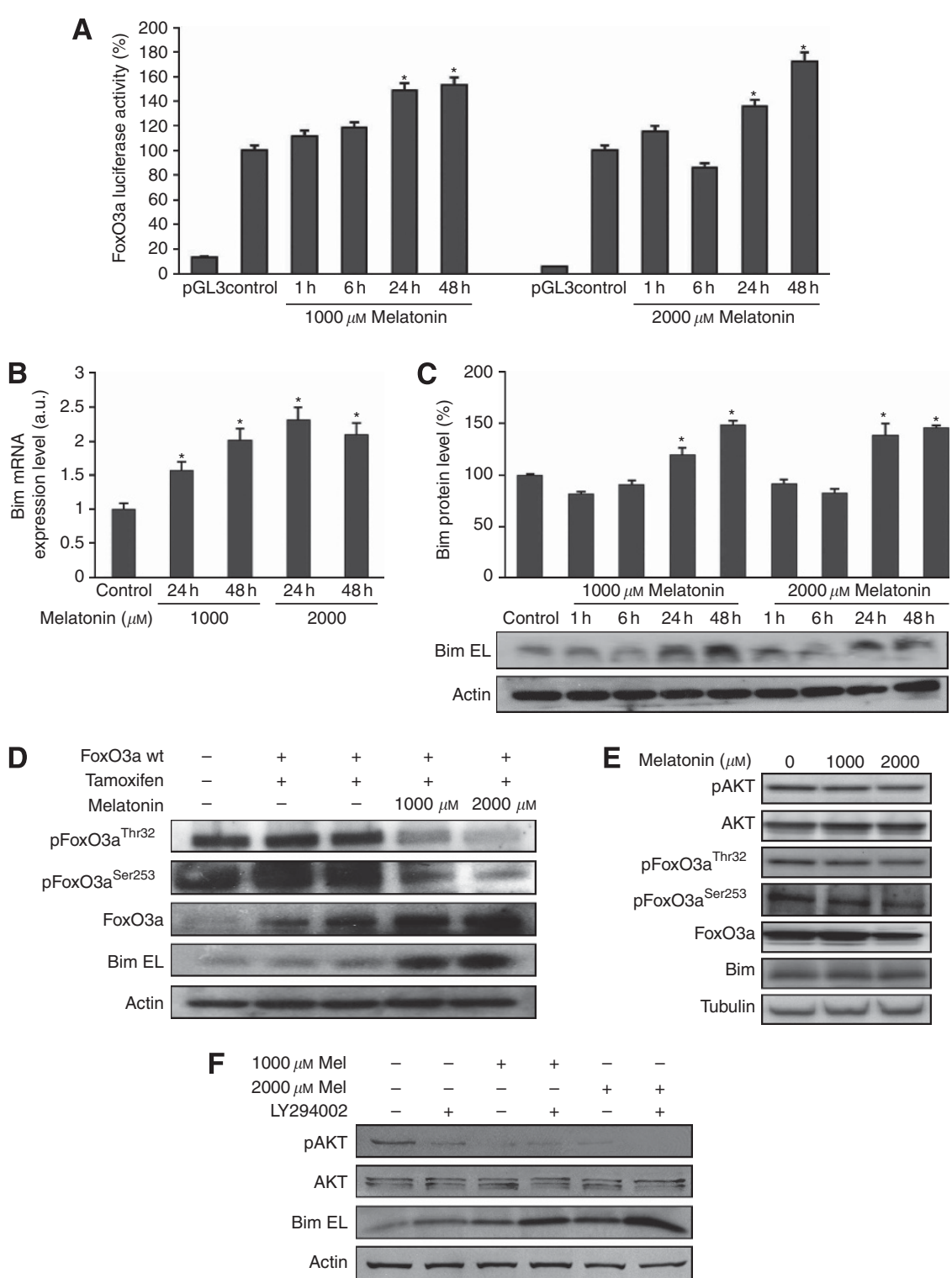

Figure 2. Consistent relation between PI3K, FoxO3a and Bim transcriptional regulation induced by melatonin administration in HepG2 cells. (A) Effect of melatonin treatment on FoxO3a luciferase activity. (B) Effect of melatonin treatment on Bim expression in HepG2 cells by real-time RT-PCR. (C) Melatonin induces the expression of Bim in time-dependent manner analysed by western blot. (D) Effect of melatonin treatment on phosphorylation status of FoxO3 and Bim EL expression. (E) Effect of melatonin treatment on PI3K/FoxO3/Bim EL pathway in primary human hepatocytes. (F) Effect of the inhibition of PI3K pathway on phospho-AKT, AKT and Bim EL expression in HepG2 cells. ${ }^{\star} P<0.05$ significant differences vs control.

RT-PCR data, demonstrating that melatonin treatment increases Bim expression both at transcriptional and translational levels (Figures 2B and C).

Effect of melatonin treatment on the PI3K/FoxO3/Bim EL pathway. The FoxO transcription factors are targets of the PI3K signalling pathway, which regulates their activity via phosphorylation on multiple threonine and serine residues. Dephosphorylation of these specific sites is associated with FoxO3a nuclear translocation, needed for its transcriptional activity (Burgering and Kops, 2002). Having observed FoxO3a transactivation of promoter elements and Bim induction by melatonin treatment, we next investigated whether Bim protein expression correlates with the phosphorylation status of FoxO3a. HepG2 cells transfected with the FoxO3a construct were treated with 1000 and $2000 \mu \mathrm{m}$ melatonin for $24 \mathrm{~h}$. Immunobloting assays showed a reduction of the dephosphorylated forms of FoxO3a at the critical phosphorylation sites ( $\mathrm{Thr}^{32}$ and $\mathrm{Ser}^{253}$ ) after melatonin treatment. It is notable that, while melatonin induced hypophosphorylation of FoxO3a, it was also accompanied by an increase in the protein level of the FoxO3a total form and Bim EL proapoptotic protein (Figure 2D). Moreover, as shown in Figure 2E, melatonin treatment induced a decrease in AKT phosphorylation in the basal state. Using the PI3K inhibitor LY294002 in combination with melatonin, enhanced expression of Bim EL protein was observed (Figure 2E). No changes in PI3K/FoxO3/Bim EL pathway were observed in primary human hepatocytes when treated with melatonin (Figure 2F). Additionally, we examined the effect of melatonin on cell viability and the PI3K-Akt pathway when stimulated by EGF. As shown in Figures $3 \mathrm{~A}$ and $\mathrm{B}$ melatonin treatment led to decreased Akt phosphorylation and cell viability, suggesting a consistent relation between PI3K, FoxO3a and Bim transcriptional upregulation.

Induction of FoxO3a nuclear translocation and Bim promoter occupancy after melatonin treatment. As melatonin treatment 

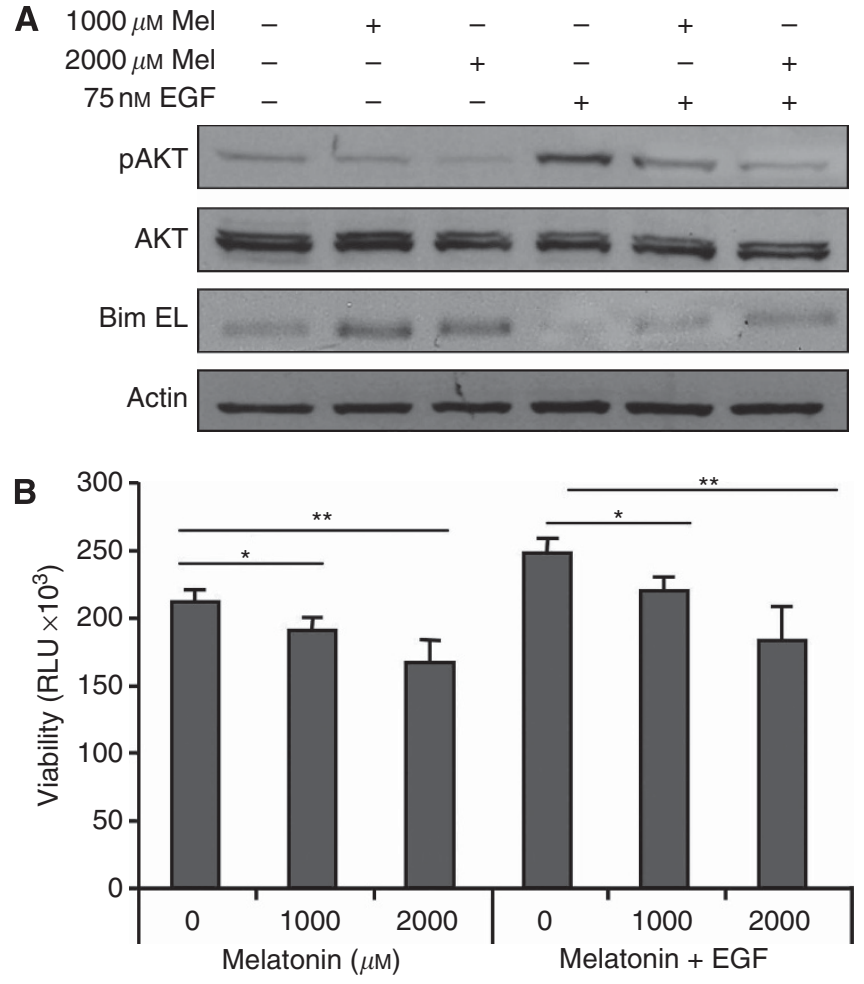

Figure 3. Melatonin is effective in cells stimulated with EGF. (A) Effect of melatonin on PI3K-Akt pathway stimulated by EGF. (B) Effect of melatonin on cell viability stimulated by EGF. Data are expressed as a percentage of mean values \pm s.e.m. of three experiments. ${ }^{\star} P<0.05,{ }^{\star} * P<0.01$ significant differences vs control. Abbreviations: $p=$ phosphorylated; $R L U=$ relative light unit.

enhanced FoxO3a dephosphorylation in liver cancer cells, we next examined whether changes on FoxO3a subcellular location were also induced by melatonin. By using fluorescence microscopy of HepG2 cells, we visualised the dynamic translocation of FoxO3a to the nucleus after melatonin treatment (Figure 4A). These results were consistent with FoxO3a nuclear localisation studies analysed by western blot of cytoplasmic and nuclear extracts after melatonin treatment (Figure 4B).

To explore whether FoxO3a is directly responsible for Bim induction after melatonin treatment, we performed gene silencing experiments transfecting HepG2 cells with siRNA specific for FoxO3a. As shown in Figure 4C, 1000 and $2000 \mu \mathrm{M}$ melatonin treatment for $24 \mathrm{~h}$ increased Bim EL protein level, while silencing of FoxO3a abrogated the melatonin-induced expression of Bim protein as determined by western blot. Next, we investigated whether the FoxO3a occupancy of the Bim promoter was affected by melatonin through ChIP assays (Figure 4D). Our results showed that upon melatonin treatment increased levels of FoxO3a could be detected binding to the promoter of Bim. Moreover, to functionally link FoxO3a and Bim with melatonin-induced apoptosis, we examined the effect of melatonin in HepG2 cells after FoxO3a and Bim knockdown with siRNA. As shown in Figure 4E, observed melatonin effects on cell viability were partially abolished when FoxO3a and Bim EL were silenced. Taken together, these results support a functional correlation between FoxO3a transcriptional activity and the levels of Bim expression in melatonin-induced apoptosis.

\section{DISCUSSION}

Hepatocellular carcinoma is the most common liver cancer and effective therapy is still lacking (Cornella et al, 2011). In this study, we tested the effects of pharmacological doses of melatonin, a natural compound synthesised in the pineal gland, which has been shown to inhibit growth of different tumours (Srinivasan et al, 2011). The role of melatonin in increasing apoptotic cell death in cancer has been widely documented (Martin-Renedo et al, 2008; Cabrera et al, 2010; Leja-Szpak et al, 2010; Cutando et al, 2011). However, there is a wide controversy about the melatonin oncostatic concentration; thus, while melatonin oncostatic effects have been reported in ME-180 and HELA human uterine neck cancer cells, OAW-42 ovarian cancer cells, HT-29 human colon cancer cells or CT-26 mouse colon cancer cells, at a concentration range 1000-6000 $\mu \mathrm{M}$ (Papazisis et al, 1998; Petranka et al, 1999; Farriol et al, 2000), human breast cancer MCF-7 cells or human choriocarcinoma Jar cells seem to be much more melatonin sensitive, responding to nanomolar doses (Hill and Blask, 1988; Shiu et al, 1999). In this respect, and having previously demonstrated that melatonin has antiproliferative and proapoptotic properties in an in vitro model of HCC (Carbajo-Pescador et al, $2009,2011)$, we used non-tumour primary human hepatocytes and the human liver cancer cell line HepG2 to analyse melatonin effects on the PI3K/FoxO3/Bim EL pathway. In our experiments, melatonin treatment had no negative effects on either this pathway or cell viability in human primary hepatocytes. Meanwhile, melatonin inhibited HepG2 cell viability, and the combination of the human CD95 agonistic Ab, anti-APO-1, with melatonin enhanced the growth inhibitory effect. Similarly to our results, melatonin has been shown to exhibit protective effects against doxorubicin-induced liver toxicity in rats $(\mathrm{Oz}$ and Ilhan, 2006), while synergistic effects on apoptosis induction of melatonin and doxorubicin have been reported in hepatoma cells (Fan et al, 2010), highlighting the selectivity and beneficial properties of melatonin based on the cell type and its features.

Dysregulation of apoptosis and cellular proliferation are clearly associated with the malignant HCC phenotype; therefore, advances in understanding these signalling pathways are necessary to develop an effective pharmacological therapy for this disease (Müller et al, 1997). We have previously demonstrated that melatonin oncostatic effects in liver cancer are partially mediated through the MT1 membrane receptor, modulation of cAMP and ERK activation (Carbajo-Pescador et al, 2011). However, the precise mechanisms whereby melatonin influences apoptosis remain unclear. FoxO transcription factors play an important role in tumour suppression by upregulation of proapoptotic genes, such as Bim (Zanella et al, 2010; Tzivion et al, 2011). While FoxO pathways have been extensively studied in different tumour cell lines (Roy et al, 2011; Hong et al, 2012), little is known about its role in HCC. The present data show for the first time that melatonin-dependent apoptosis in HepG2 cells may be mediated, at least in part, by FoxO3a activation and subsequently increased Bim expression.

Apoptotic cell death is a complex programme mainly controlled by the Bcl-2 family proteins. We have previously reported, using HepG2 cells, an extrinsic apoptosis induction after melatonin treatment, associated with upregulation of one of these proapoptotic proteins, Bax, cytochrome $c$ release and caspases activation (Martin-Renedo et al, 2008). However, it is known that the presence of BH3-only molecules like Bid, Bim and Puma is required for direct activation of Bax at the mitochondria. In this study, we observed an increase in Bim expression, both at mRNA and at protein levels after melatonin treatment in HepG2 cells. Upregulation and activation of Bim protein is involved in the oncostatic effect of many other chemotherapeutic drugs in liver cancer, pointing to its role in critical steps of the apoptosis initiation (Schneider-Jakob et al, 2010). It has been reported that Bid, Bim and Puma triple-knockout mice present developmental defects associated with deficiency of Bax. Moreover, genetic deletion in neurons and $\mathrm{T}$ lymphocytes prevents the 

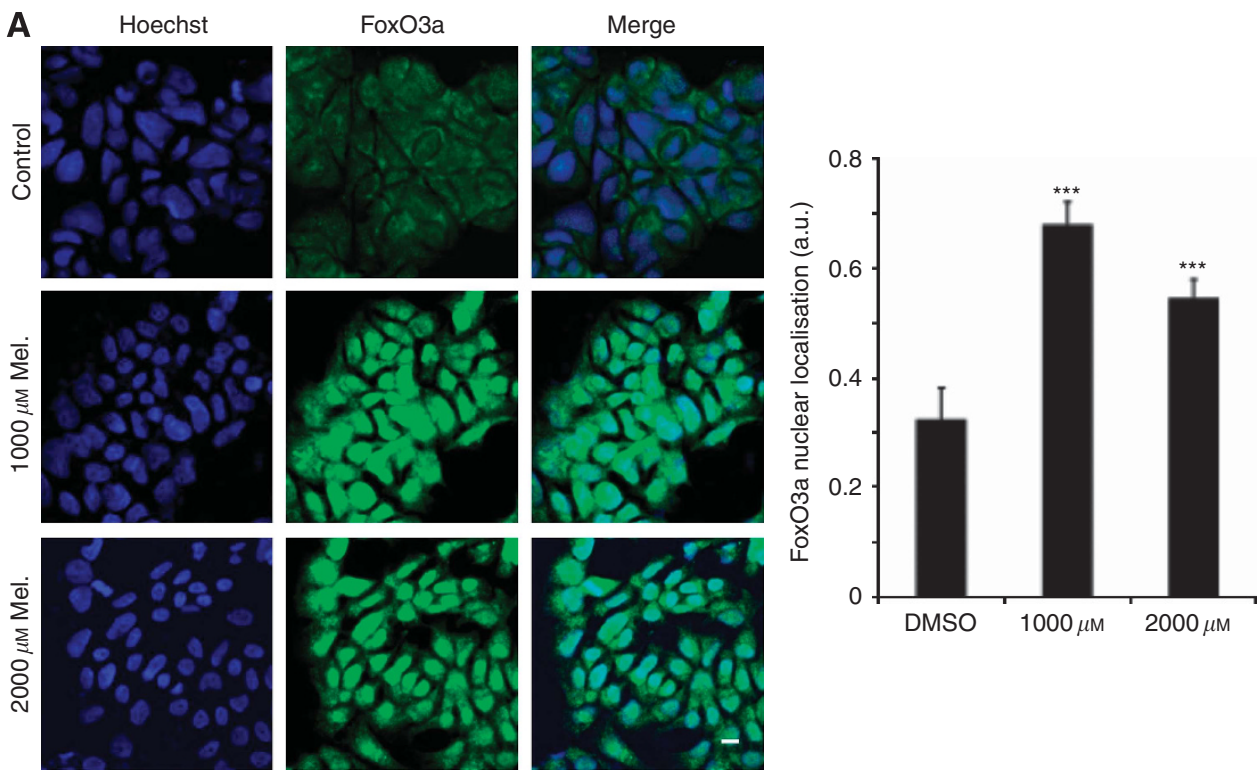

B
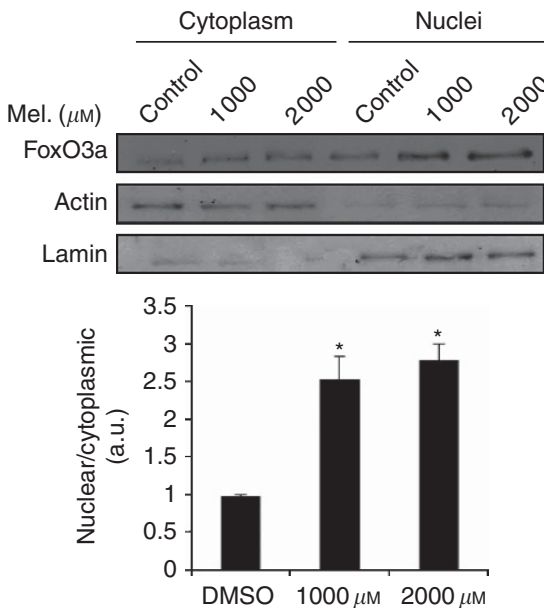

D

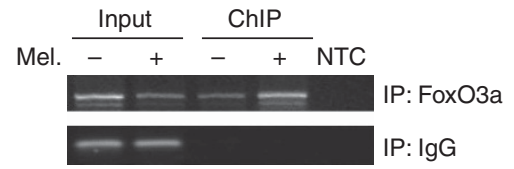

C $1000 \mu \mathrm{m} \mathrm{Mel.}$ $2000 \mu \mathrm{m}$ Mel. siRNA contro SiRNA FoxO3a

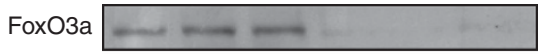

Bim EL

Actin

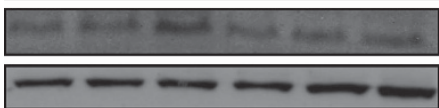

E

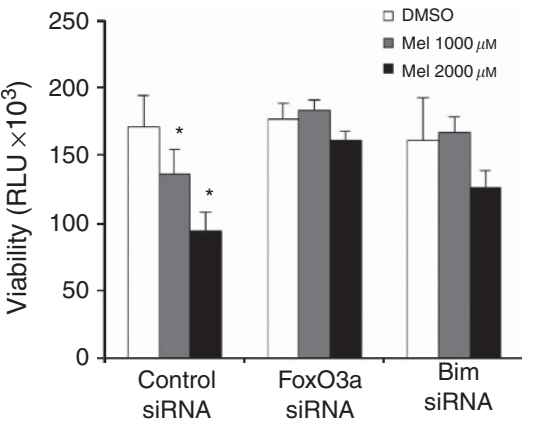

Figure 4. Induction of FoxO3a nuclear translocation and Bim promoter occupancy after melatonin treatment. (A) FoxO3a nuclear translocation. $\star \star \star P<0.001$ significant differences in nuclear localisation of FoxO3a in control vs melatonin-treated cells. Data points represent mean \pm s.d. from separate high-power field images. Bar $=10 \mu \mathrm{m}$. (B) Effect of melatonin on FoxO3a cytoplasmic and nuclear protein expression. Lower panel is nuclear to cytoplasmic ratios of FoxO3a of western blot samples. (C) Effect of FoxO3a silencing and melatonin treatment on Bim expression. (D) Melatonin enhances binding of FoxO3a to Bim promoter region as analysed by ChIP. (E) Effect of FoxO3a and Bim silencing on HepG2 cell viability. Representative results of three individual experiments. ${ }^{\star} P<0.05$. Abbreviations: $a . u$. $=$ arbitrary units; IgG $=$ rabbit control immunoglobulin G; NTC= non-template control; RLU = relative light unit.

homo-oligomerisation of Bax and Bad, and thereby cytochrome $c$ mediated activation of caspases in response to diverse death signals (Ren et al, 2010).

Although we observed an induction of Bim expression both at RNA and at protein level after melatonin treatment, FoxO3a transcriptional activity was also significantly increased from 1 to $48 \mathrm{~h}$ with 1000 and $2000 \mu \mathrm{m}$ melatonin incubation, suggesting a possible correlation between FoxO3a as a transcription factor of Bim in HepG2 liver cancer cells. Moreover, further experiments focused on understanding the molecular mechanism that underlay FoxO3a activation, showed a decrease in the dephosphorylated forms of FoxO3a at $\mathrm{Thr}^{32}$ and $\mathrm{Ser}^{253}$, complemented by an increase of total FoxO3a and Bim EL in response to melatonin treatment.
FoxO3a accumulation and Bim protein expression were greatly reduced upon silencing of FoxO3a, providing evidences to suggest that FoxO3a is functioning as a transcriptional regulator of Bim expression in HepG2 cells after melatonin treatment. It has been previously demonstrated that FoxO3a pathway can induce Bim expression and subsequently cell death in several cancer models, like MCF-7 breast cancer cell line (Sharma et al, 2011), mice xenografts model of pancreas tumour (Boreddy et al, 2011) and lymphoma cells (Bhalla et al, 2011), among others. It is generally accepted that the FoxO family is a key downstream target of the PI3K pathway (Weidinger et al, 2011). While phosphorylation of FoxO factors by Akt causes relocalisation of FoxO proteins from the nucleus to the cytoplasm, dephosphorylated FoxO forms 
activate target genes (Hong et al, 2012). Therefore, our work provides clear evidence of melatonin-induced activation of FoxO3 in cells, promoting changes in its phosphorylation status. Moreover, translocation of FoxO3a to the nucleus was also confirmed by fluorescence microscopy experiments as well as by FoxO3a western blot in nuclear and cytoplasmic extracts. In this study, melatonin caused an inhibition of AKT phosphorylation even after EGF stimulation, and the PI3K inhibitor LY294002, combined with melatonin, resulted in a synergic effect enhancing Bim protein expression. Our knowledge of the mechanisms by which melatonin induces apoptosis in human HepG2 hepatoma cells are limited; however, the FoxO3a/Bim pathway has been shown to participate in apoptotic processes in response to other chemotherapeutic agents like cisplatin (Fernandez de Mattos et al, 2008; Yuan et al, 2011). Moreover, resveratrol, another antioxidant molecule, has been reported to behave as melatonin, exerting an oncostatic and proapoptotic activity in different tumour cells, including HepG2 (Hsieh et al, 2005; Notas et al, 2006). Although little is known about the resveratrol effect on the FoxO3a pathway, several groups have reported FoxO3a dephosphorylation, nuclear translocation and Bim induction after resveratrol treatment in in vitro cancer models (Chen et al, 2010; Roy et al, 2011), helping us to support our hypothesis.

Our study provides important information regarding the mechanisms by which melatonin regulates apoptosis through the activation of FoxO transcription factors. Taken together, all these results demonstrate that Bim plays a significant role in melatonininduced apoptosis in HepG2 liver cancer cells, most likely through the activity of FoxO3a. Thereby, while this work could represent a significant advance for the understanding of the melatonin oncostatic pathway in vitro, further in vivo experiments are required to bridge the gap between clinical applications and to investigate whether this indol could be safely used as a therapeutic drug in HCC treatment, perhaps as an adjuvant.

\section{ACKNOWLEDGEMENTS}

Sara Carbajo-Pescador is granted by the Consejería de Educación (Junta de Castilla y León, Spain) and Fondo Social Europeo. CIBERehd is funded by Instituto de Salud Carlos III. This work has been partially supported by Junta de Castilla y León (ref. LE117A11-2), and Fundación Investigación Sanitaria en León and the Forschungszentrum Immunologie (FZI), Mainz.

\section{CONFLICT OF INTEREST}

The authors declare no conflict of interest.

\section{REFERENCES}

Anton S, Melville L, Rena G (2007) Epigallocatechin gallate (EGCG) mimics insulin action on the transcription factor FOXO1a and elicits cellular responses in the presence and absence of insulin. Cell Signal 19: 378-383.

Barthel A, Schmoll D, Unterman TG (2005) FoxO proteins in insulin action and metabolism. Trends Endocrinol Metab 16: 183-189.

Bhalla S, Evens AM, Dai B, Prachand S, Gordon LI, Gartenhaus RB (2011) The novel anti-MEK small molecule AZD6244 induces BIM-dependent and AKT-independent apoptosis in diffuse large B-cell lymphoma. Blood 118: 1052-1061.

Boreddy SR, Pramanik KC, Srivastava SK (2011) Pancreatic tumour suppression by benzyl isothiocyanate is associated with inhibition of PI3K/ AKT/FOXO pathway. Clin Cancer Res 17: 1784-1795.

Brunet A, Bonni A, Zigmond MJ, Lin MZ, Juo P, Hu LS, Anderson MJ, Arden KC, Blenis J, Greenberg ME (1999) Akt promotes cell survival by phosphorylating and inhibiting a forkhead transcription factor. Cell 96: 857-868.

Burgering BM, Kops GJ (2002) Cell cycle and death control: long live forkheads. Trends Biochem Sci 27: 352-360.

Cabrera J, Negrin G, Estevez F, Loro J, Reiter RJ, Quintana J (2010) Melatonin decreases cell proliferation and induces melanogenesis in human melanoma SK-MEL-1 cells. J Pineal Res 49: 45-54.

Calnan DR, Brunet A (2008) The FoxO code. Oncogene 27: 2276-2288.

Carbajo-Pescador S, Garcia-Palomo A, Martin-Renedo J, Piva M, GonzalezGallego J, Mauriz JL (2011) Melatonin modulation of intracellular signaling pathways in hepatocarcinoma HepG2 cell line: role of the MT1 receptor. J Pineal Res 51: 463-471.

Carbajo-Pescador S, Martin-Renedo J, Garcia-Palomo A, Tunon MJ, Mauriz JL, Gonzalez-Gallego J (2009) Changes in the expression of melatonin receptors induced by melatonin treatment in hepatocarcinoma HepG2 cells. J Pineal Res 47: 330-338.

Chen Q, Ganapathy S, Singh KP, Shankar S, Srivastava RK (2010) Resveratrol induces growth arrest and apoptosis through activation of FOXO transcription factors in prostate cancer cells. PLoS One 5: e15288.

Chiu CC, Chen JY, Lin KL, Huang CJ, Lee JC, Chen BH, Chen WY, Lo YH, Chen YL, Tseng CH, Chen YL, Lin SR (2010) p38 MAPK and NF-kappaB pathways are involved in naphtho[1,2-b] furan-4,5-dione induced antiproliferation and apoptosis of human hepatoma cells. Cancer Lett 295: 92-99.

Cini G, Neri B, Pacini A, Cesati V, Sassoli C, Quattrone S, D’Apolito M, Fazio A, Scapagnini G, Provenzani A, Quattrone A (2005) Antiproliferative activity of melatonin by transcriptional inhibition of cyclin D1 expression: a molecular basis for melatonin-induced oncostatic effects. J Pineal Res 39: $12-20$.

Cornella H, Alsinet C, Villanueva A (2011) Molecular Pathogenesis of Hepatocellular Carcinoma. Alcohol Clin Exp Res 35: 821-825.

Cutando A, Aneiros-Fernandez J, Aneiros-Cachaza J, Arias-Santiago S (2011) Melatonin and cancer: current knowledge and its application to oral cavity tumours. J Oral Pathol Med 40: 593-597.

Denizot F, Lang R (1986) Rapid colorimetric assay for cell growth and survival. Modifications to the tetrazolium dye procedure giving improved sensitivity and reliability. J Immunol Methods 89: 271-277.

Dijkers PF, Medema RH, Lammers JW, Koenderman L, Coffer PJ (2000) Expression of the pro-apoptotic Bcl-2 family member Bim is regulated by the forkhead transcription factor FKHR-L1. Curr Biol 10: 1201-1204.

El-Serag HB (2011) Hepatocellular carcinoma. N Engl J Med 365: 1118-1127. Ewings KE, Wiggins CM, Cook SJ (2007) Bim and the pro-survival Bcl-2 proteins: opposites attract, ERK repels. Cell Cycle 6: 2236-2240.

Fan LL, Sun GP, Wei W, Wang ZG, Ge L, Fu WZ, Wang H (2010) Melatonin and doxorubicin synergistically induce cell apoptosis in human hepatoma cell lines. World J Gastroenterol 16: 1473-1481.

Farriol M, Venereo Y, Orta X, Castellanos JM, Segovia-Silvestre T (2000) In vitro effects of melatonin on cell proliferation in a colon adenocarcinoma line. J Appl Toxicol 20: 21-24.

Fei M, Zhao Y, Wang Y, Lu M, Cheng C, Huang X, Zhang D, Lu J, He S, Shen A (2009) Low expression of Foxo3a is associated with poor prognosis in ovarian cancer patients. Cancer Invest 27: 52-59.

Fernandez de Mattos S, Villalonga P, Clardy J, Lam EW (2008) FOXO3a mediates the cytotoxic effects of cisplatin in colon cancer cells. Mol Cancer Ther 7: 3237-3246.

Futagami M, Sato S, Sakamoto T, Yokoyama Y, Saito Y (2001) Effects of melatonin on the proliferation and cis-diamminedichloroplatinum (CDDP) sensitivity of cultured human ovarian cancer cells. Gynecol Oncol 82: $544-549$.

Garcia-Navarro A, Gonzalez-Puga C, Escames G, Lopez LC, Lopez A, LopezCantarero M, Camacho E, Espinosa A, Gallo MA, Acuna-Castroviejo D (2007) Cellular mechanisms involved in the melatonin inhibition of HT-29 human colon cancer cell proliferation in culture. J Pineal Res 43: 195-205.

Garcia-Santos G, Antolin I, Herrera F, Martin V, Rodriguez-Blanco J, del Pilar Carrera M, Rodriguez C (2006) Melatonin induces apoptosis in human neuroblastoma cancer cells. J Pineal Res 41: 130-135.

Gonzalez A, Del Castillo-Vaquero A, Miro-Moran A, Tapia JA, Salido GM (2010) Melatonin reduces pancreatic tumour cell viability by altering mitochondrial physiology. J Pineal Res 50: 250-260.

Hill SM, Blask DE (1988) Effects of the pineal hormone melatonin on the proliferation and morphological characteristics of human breast cancer cells (MCF-7) in culture. Cancer Res 48: 6121-6126. 
Hong ZY, Lee HJ, Shin DY, Kim SK, Seo M, Lee EJ (2012) Inhibition of Akt/ FOXO3a signaling by constitutively active FOXO3a suppresses growth of follicular thyroid cancer cell lines. Cancer Lett 314: 34-40.

Hsieh TC, Wang Z, Hamby CV, Wu JM (2005) Inhibition of melanoma cell proliferation by resveratrol is correlated with upregulation of quinone reductase 2 and p53. Biochem Biophys Res Commun 334: 223-230.

Jemal A, Bray F, Center MM, Ferlay J, Ward E, Forman D (2011) Global cancer statistics. CA Cancer J Clin 61: 69-90.

Jin GS, Kondo E, Miyake T, Shibata M, Takashima T, Liu YX, Hayashi K, Akagi T, Yoshino T (2004) Expression and intracellular localization of FKHRL1 in mammary gland neoplasms. Acta Med Okayama 58: 197-205.

Leja-Szpak A, Jaworek J, Pierzchalski P, Reiter RJ (2010) Melatonin induces pro-apoptotic signaling pathway in human pancreatic carcinoma cells (PANC-1). J Pineal Res 49: 248-255.

Lin K, Dorman JB, Rodan A, Kenyon C (1997) Daf-16: An HNF-3/forkhead family member that can function to double the life-span of Caenorhabditis elegans. Science 278: 1319-1322.

Martin-Renedo J, Mauriz JL, Jorquera F, Ruiz-Andres O, Gonzalez P, Gonzalez-Gallego J (2008) Melatonin induces cell cycle arrest and apoptosis in hepatocarcinoma HepG2 cell line. J Pineal Res 45: $532-540$.

Müller M, Strand S, Hug H, Heinemann EM, Walczak H, Hofmann WJ, Stremmel W, Krammer PH, Galle PR (1997) Drug-induced apoptosis in hepatoma cells is mediated by the CD95 (APO-1/Fas) receptor/ligand system and involves activation of wild-type p53. J Clin Invest 99: 403-413.

Notas G, Nifli AP, Kampa M, Vercauteren J, Kouroumalis E, Castanas E (2006) Resveratrol exerts its antiproliferative effect on HepG2 hepatocellular carcinoma cells, by inducing cell cycle arrest, and NOS activation. Biochim Biophys Acta 1760: 1657-1666.

Oz E, Ilhan MN (2006) Effects of melatonin in reducing the toxic effects of doxorubicin. Mol Cell Biochem 286: 11-15.

Pan M, Song YL, Xu JM, Gan HZ (2006) Melatonin ameliorates nonalcoholic fatty liver induced by high-fat diet in rats. J Pineal Res 41: 79-84.

Papazisis KT, Kouretas D, Geromichalos GD, Sivridis E, Tsekreli OK, Dimitriadis KA, Kortsaris AH (1998) Effects of melatonin on proliferation of cancer cell lines. J Pineal Res 25: 211-218.

Paroni G, Fratelli M, Gardini G, Bassano C, Flora M, Zanetti A, Guarnaccia V, Ubezio P, Centritto F, Terao M, Garattini E (2011) Synergistic antitumor activity of lapatinib and retinoids on a novel subtype of breast cancer with coamplification of ERBB2 and RARA. Oncogene.

Petranka J, Baldwin W, Biermann J, Jayadev S, Barrett JC, Murphy E (1999) The oncostatic action of melatonin in an ovarian carcinoma cell line. J Pineal Res 26: 129-136.

Ren D, Tu HC, Kim H, Wang GX, Bean GR, Takeuchi O, Jeffers JR, Zambetti GP, Hsieh JJ, Cheng EH (2010) BID, BIM, and PUMA are essential for activation of the BAX- and BAK-dependent cell death program. Science 330: $1390-1393$.

Rena G, Woods YL, Prescott AR, Peggie M, Unterman TG, Williams MR, Cohen P (2002) Two novel phosphorylation sites on FKHR that are critical for its nuclear exclusion. EMBO J 21: 2263-2271.

Roy SK, Chen Q, Fu J, Shankar S, Srivastava RK (2011) Resveratrol inhibits growth of orthotopic pancreatic tumours through activation of FOXO transcription factors. PLoS One 6: e25166.

Schneider-Jakob S, Corazza N, Badmann A, Sidler D, Stuber-Roos R, Keogh A, Frese S, Tschan M, Brunner T (2010) Synergistic induction of cell death in liver tumour cells by TRAIL and chemotherapeutic drugs via the BH3only proteins Bim and Bid. Cell Death Dis 1: e86.

Sharma G, Kar S, Palit S, Das PK (2011) 18Beta-glycyrrhetinic acid induces apoptosis through modulation of Akt/FOXO3a/Bim pathway in human breast cancer MCF-7 cells. J Cell Physiol 227: 1923-1931.

Shiu SY, Li L, Xu JN, Pang CS, Wong JT, Pang SF (1999) Melatonin-induced inhibition of proliferation and G1/S cell cycle transition delay of human choriocarcinoma JAr cells: possible involvement of MT2 (MEL1B) receptor. J Pineal Res 27: 183-192.

Srinivasan V, Pandi-Perumal SR, Brzezinski A, Bhatnagar KP, Cardinali DP (2011) Melatonin, immune function and cancer. Recent Pat Endocr Metab Immune Drug Discov 5: 109-123.

Su JL, Cheng X, Yamaguchi H, Chang YW, Hou CF, Lee DF, Ko HW, Hua KT, Wang YN, Hsiao M, Chen PB, Hsu JM, Bast Jr RC, Hortobagyi GN, Hung MC (2011) FOXO3a-dependent mechanism of E1A-induced chemosensitization. Cancer Res 71: 6878-6887.

Subramanian P, Mirunalini S, Pandi-Perumal SR, Trakht I, Cardinali DP (2007) Melatonin treatment improves the antioxidant status and decreases lipid content in brain and liver of rats. Eur J Pharmacol 571: 116-119.

Sunters A, Fernandez de Mattos S, Stahl M, Brosens JJ, Zoumpoulidou G, Saunders CA, Coffer PJ, Medema RH, Coombes RC, Lam EW (2003) FoxO3a transcriptional regulation of Bim controls apoptosis in paclitaxeltreated breast cancer cell lines. J Biol Chem 278: 49795-49805.

Tahan V, Atug O, Akin H, Eren F, Tahan G, Tarcin O, Uzun H, Ozdogan O, Tarcin O, Imeryuz N, Ozguner F, Celikel C, Avsar E, Tozun N (2009) Melatonin ameliorates methionine- and choline-deficient diet-induced nonalcoholic steatohepatitis in rats. J Pineal Res 46: 401-407.

Thong-Ngam D, Samuhasaneeto S, Kulaputana O, Klaikeaw N (2007) Nacetylcysteine attenuates oxidative stress and liver pathology in rats with non-alcoholic steatohepatitis. World J Gastroenterol 13: 5127-5132.

Tran H, Brunet A, Grenier JM, Datta SR, Fornace Jr AJ, DiStefano PS, Chiang LW, Greenberg ME (2002) DNA repair pathway stimulated by the forkhead transcription factor FOXO3a through the Gadd45 protein. Science 296: 530-534.

Tzivion G, Dobson M, Ramakrishnan G (2011) FoxO transcription factors: regulation by AKT and 14-3-3 proteins. Biochim Biophys Acta 1813: 1938-1945.

Weidinger C, Krause K, Mueller K, Klagge A, Fuhrer D (2011) FOXO3 is inhibited by oncogenic PI3K/Akt signaling but can be reactivated by the NSAID sulindac sulfide. J Clin Endocrinol Metab 96: E1361-E1371.

Yang JY, Xia W, Hu MC (2006) Ionizing radiation activates expression of FOXO3a, Fas ligand, and Bim, and induces cell apoptosis. Int J Oncol 29: 643-648.

Yuan Z, Wang F, Zhao Z, Zhao X, Qiu J, Nie C, Wei Y (2011) BIM-mediated AKT phosphorylation is a key modulator of arsenic trioxide-induced apoptosis in cisplatin-sensitive and -resistant ovarian cancer cells PLoS One 6: e20586.

Zanella F, Link W, Carnero A (2010) Understanding FOXO, new views on old transcription factors. Curr Cancer Drug Targets 10: 135-146.

This work is published under the standard license to publish agreement. After 12 months the work will become freely available and the license terms will switch to a Creative Commons AttributionNonCommercial-Share Alike 3.0 Unported License. 